\title{
Compressible primitive equations: formal derivation and stability of weak solutions
}

\author{
Mehmet Ersoy ${ }^{\mathrm{a}, \mathrm{b}}$, Timack Ngom ${ }^{\mathrm{a}, \mathrm{c}}$, Mamadou $\mathrm{Sy}^{\mathrm{c}}$ \\ ${ }^{a}$ LAMA, UMR 5127 CNRS, Université de Savoie, 73376 Le Bourget du lac cedex, France. \\ ${ }^{b}$ BCAM - Basque Center for Applied Mathematics, Bizkaia Technology Park 500, 48160, \\ Derio, Basque Country, Spain. \\ ${ }^{c}$ Laboratoire d'Analyse Numérique et Informatique(LANI), Université Gaston Berger de \\ Saint-Louis, UFR SAT BP 234 Saint-Louis, Sénégal.
}

\begin{abstract}
We present a formal derivation of Compressible Primitive Equations (CPEs) for atmosphere modeling. They are obtained from the 3-D compressible NavierStokes equations with an anisotropic viscous stress tensor depending on the density. Then, we study the stability of weak solutions to this problem by introducing an intermediate model obtained by a suitable change of variables. This intermediate model is more simpler and practical to achieve the main result.
\end{abstract}

Keywords: Compressible primitive equations, Compressible viscous fluid, Anisotropic viscous tensor, A priori estimates, Stability of weak solutions.

\section{Contents}

1 Introduction 2

2 Formal derivation of the atmosphere model 4

2.1 Formal derivation of the CPEs $\ldots \ldots \ldots \ldots \ldots$

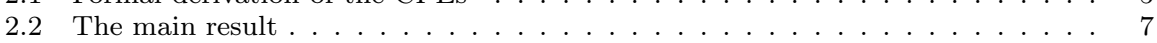

3 Stability of weak solutions for the CPEs $\quad 8$

3.1 A model problem; an intermediate model . . . . . . . . . . . . . . . . . . . 9

3.2 Mathematical study of the model problem . . . . . . . . . . . . . . . 9

3.2.1 Energy and entropy estimates . . . . . . . . . . . . . . 10

3.2 .2 Convergence of $\sqrt{\xi_{n}} \ldots \ldots \ldots \ldots \ldots \ldots$

3.2.3 Bounds of $\sqrt{\xi_{n}} \mathbf{u}_{n}$ and $\sqrt{\xi_{n}} w_{n} \ldots \ldots \ldots \ldots \ldots$

3.2 .4 Convergence of $\xi_{n} \mathbf{u}_{n} \ldots \ldots \ldots \ldots \ldots \ldots$

3.2 .5 Convergence of $\sqrt{\xi_{n}} \mathbf{u}_{n}$ and $\xi_{n} w_{n} \ldots \ldots \ldots \ldots \ldots \ldots$

3.2 .6 Convergence step . . . . . . . . . . . . . . . . . . . . . . . . . . 19

3.2 .7 Proof of Theorem $1 \ldots \ldots \ldots \ldots$

Email addresses: Mehmet.Ersoy@univ-savoie.fr (Mehmet Ersoy),

Timack.ngom@etu.univ-savoie.fr (Timack Ngom), syndioum@yahoo.fr (Mamadou Sy) 


\section{Introduction}

Among equations of geophysical fluid dynamics (see Buntebarth [5]), classically the equations governing the motion of the atmosphere are the Primitive Equations (PEs). In the hierarchy of geophysical fluid dynamics models, they are situated between non hydrostatic models and shallow water models.

\section{Derivation of the Compressible PEs}

CPEs are obtained from the hydrostatic approximation (see, for instance, Pedlowski [11] or Temam et al. [12]) of the full 3 dimensional set of NavierStokes equations for atmosphere modeling. Neglecting phenomena such as the evaporation and solar heating, the Primitive Equations read:

$$
\left\{\begin{array}{l}
\frac{d}{d t} \rho+\rho \operatorname{div} \mathbf{U}=0 \\
\rho \frac{d}{d t} \mathbf{u}+\nabla_{x} p=\mathcal{D} \\
\partial_{y} p=-g \rho \\
p(\rho)=c^{2} \rho
\end{array}\right.
$$

where

$$
\frac{d}{d t}=\partial_{t}+\mathbf{u} \cdot \nabla_{x}+v \partial_{y}
$$

with $x=\left(x_{1}, x_{2}\right)$ the horizontal and $y$ the vertical coordinate.

$\mathbf{U}$ is the three dimensional velocity vector with component $\mathbf{u}=\left(u_{1}, u_{2}\right)$ for the horizontal velocity and $v$ for the vertical one. The terms $\rho, p, \mathbf{g}$ stand for the density, the barotropic pressure and the gravity vector $(0,0, g)$. The constant $c^{2}$ is usually set to $\mathcal{R} \mathcal{T}$ where $\mathcal{R}$ is the specific gas constant for the air and $\mathcal{T}$ the temperature.

In the present paper, the diffusion term $\mathcal{D}$ reads:

$$
\mathcal{D}=2 \operatorname{div}_{x}\left(\nu_{1}(t, x, y) D_{x}(\mathbf{u})\right)+\partial_{y}\left(\nu_{2}(t, x, y) \partial_{y} \mathbf{u}\right) .
$$

It is obtained by introducing an anisotropic viscous tensor in the initial NavierStokes equations where $\operatorname{div}_{x}$ stands for $\partial_{x_{1}}+\partial_{x_{2}}, D_{x}=\left(\nabla_{x}+\nabla_{x}^{t}\right) / 2$ and $\nu_{1}(t, x, y) \neq \nu_{2}(t, x, y)$ represent the anisotropic pair of viscosity depending on the density $\rho$.

The main difference with respect to the classical viscous term found in the litterature (see for instance Temam et al. [12]) is that viscosities depend on the density.

\section{Mathematical analysis of CPEs}

The mathematical analysis of PEs for atmosphere modeling was first carried out by Lions et al. [9]. These authors have taken into account evaporation and solar heating with constant viscosities. They produced the mathematical formulation in 2 and 3 dimensions based on the works of J. Leray and obtained 
the existence of weak solutions for all time (see also Temam et al. [12] where the result was proved by different means).

Following Temam et al. [12], Ersoy et al. [6] showed the global weak existence for the 2-D version of model (1) by a useful change of vertical coordinates.

Currently, up to our knowledge, there is no way to prove an existence or stability result for Model (1). One of the difficulties encountered is to obtain energy estimates. Indeed, proceeding by standard techniques, multiplying the conservation of the momentum equations of System (1) by (u, $v)$, we get:

$\frac{d}{d t} \int_{\Omega}\left(\rho|u|^{2}+\rho \ln \rho-\rho+1\right) d x d y+\int_{\Omega} 2 \nu_{1}\left|D_{x}(u)\right|^{2}+\nu_{2}\left|\partial_{y}^{2} u\right| d x d y+\int_{\Omega} \rho g v d x d y$

where the sign of the integral $\int_{\Omega} \rho g v d x d y$ is unknown. There is no way to control, prima facie, the integral term $\int_{\Omega} \rho g v d x$ introduced by the hydrostatic equation $\partial_{y} p=-g \rho$. To overcome this problem, we make a change of variables and we study an intermediate problem. Following Ersoy et al. [6], setting

$$
z=1-e^{-g / c^{2} y} \text { and } w(t, x, z)=e^{-g / c^{2} y} v(t, x, y)
$$

and assuming

$$
\nu_{1}(t, x, y)=\bar{\nu}_{1} \rho(t, x, y) \text { and } \nu_{2}(t, x, y)=\bar{\nu}_{2} \rho(t, x, y) e^{2 y} \text { with } \bar{\nu}_{i}>0,
$$

we obtain the following model:

$$
\left\{\begin{array}{l}
\frac{d}{d t} \xi+\xi\left(\operatorname{div}_{x} \mathbf{u}+\partial_{z} w\right)=0 \\
\rho \frac{d}{d t} \mathbf{u}+\nabla_{x} p=\mathcal{D}_{z} \\
\partial_{z} \xi=0 \\
p(\xi)=c^{2} \xi
\end{array}\right.
$$

where $\frac{d}{d t}$ denotes

$$
\frac{d}{d t}=\partial_{t}+\mathbf{u} \cdot \nabla_{x}+w \partial_{z}
$$

and

$$
\mathcal{D}_{z}=2 \operatorname{div}_{x}\left(\nu_{1}(t, x, z) D_{x}(\mathbf{u})\right)+\partial_{z}\left(\nu_{2}(t, x, z) \partial_{z} \mathbf{u}\right) .
$$

Consequently, in the computation of the energy the integral term vanishes since the right hand side of the equation $\partial_{z} \xi$ becomes 0 . Thus, we can obtain preliminary estimates.

In order to show the weak stability, the additional required estimates are provided by the BD-entropy (see, for instance, Bresch $[2,4,3,1]$ ) by adding a regularizing term to Equations (2). In this paper, we have added a quadratic friction source term. Combining this term to the viscous one (3) brings regularity on the density which is required to pass to the limit in the non linear 
terms (e.g. for the term $\xi \mathbf{u} \otimes \mathbf{u}$ where typically a strong convergence of $\sqrt{\xi} \mathbf{u}$ is needed). Finally, energy and BD-entropy estimates are enough to show a weak stability result for Model (2) and by the reverse change of variables for Model (1).

Currently, the question of existence of weak solutions remains an open question for Model (2) (so, also for the Model (1)).

This paper is organized as follows. In Section 2, starting from the 3-D compressible Navier-Stokes equations with an anisotropic viscous tensor, we formally derive the Model (1). Then, we present the main result in Section 2.2. We provide a complete proof in Section 3.2.

\section{Formal derivation of the atmosphere model}

We consider the Navier-Stokes model in a bounded three dimensional domain with periodic boundary conditions on $\Omega_{x}$ and free conditions on the rest of the boundary. More exactly, we assume that the motion of the medium occurs in a domain $\Omega=\left\{(x, y) ; x \in \Omega_{x}, 0<y<H\right\}$ where $\Omega_{x}=\mathbb{T}^{2}$ is the bi-dimensional torus and $H$ the characteristic scale of the altitude. The full Navier-Stokes equations are:

$$
\begin{array}{r}
\partial_{t} \rho+\operatorname{div}(\rho u)=0, \\
\partial_{t}(\rho u)+\operatorname{div}(\rho u \otimes u)-\operatorname{div} \sigma-\rho f=0, \\
p=p(\rho)
\end{array}
$$

where $\rho$ is the density of the fluid and $u=(\mathbf{u}, v)^{t}$ stands for the fluid velocity with $\mathbf{u}=\left(u_{1}, u_{2}\right)^{t}$ the horizontal component and $v$ the vertical one. $\sigma$ is the total asymmetric stress tensor. The pressure law is given by the equation of state:

$$
p(\rho)=c^{2} \rho
$$

for some given positive constant $c$. The term $f$ regroups the quadratic friction source term and the gravity strength:

$$
f=-R \sqrt{u_{1}^{2}+u_{2}^{2}}\left(u_{1}, u_{2}, 0\right)^{t}-g \mathbf{k}
$$

where $R$ is a positive constant, $g$ is the gravitational constant and $\mathbf{k}=(0,0,1)^{t}$ (where $X^{t}$ stands for the transpose of tensor $X$ ).

Remark 1. As we will see later, the friction term is a mathematical remedy to ensure the stability of weak solutions of the problem.

The total stress tensor is:

$$
\sigma=-p I_{3}+2 \Sigma . D(u)+\lambda \operatorname{div}(u) I_{3}
$$

where the term $\Sigma . D(u)$ reads:

$$
\left(\begin{array}{cc}
2 \mu_{1} D_{x}(\mathbf{u}) & \mu_{2}\left(\partial_{y} \mathbf{u}+\nabla_{x} v\right) \\
\mu_{3}\left(\partial_{y} \mathbf{u}+\nabla_{x} v\right)^{t} & 2 \mu_{3} \partial_{y} v
\end{array}\right)
$$


with $I_{3}$ the identity matrix. In the definition above, the term $\Sigma=\Sigma(t, x, y)$ stands for the following non constant anisotropic viscous tensor (see, for instance, $[8,7,6])$ :

$$
\left(\begin{array}{lll}
\mu_{1} & \mu_{1} & \mu_{2} \\
\mu_{1} & \mu_{1} & \mu_{2} \\
\mu_{3} & \mu_{3} & \mu_{3}
\end{array}\right) .
$$

The term $D_{x}(\mathbf{u})$ is the strain tensor with respect to the horizontal variable $x$, i.e.

$$
2 D_{x}(\mathbf{u})=\nabla_{x} \mathbf{u}+\nabla_{x}^{t} \mathbf{u}=\left(\partial_{x_{i}} \mathbf{u}_{j}+\partial_{x_{j}} \mathbf{u}_{i}\right)_{1 \leqslant i, j \leqslant 2} .
$$

The last term $\lambda \operatorname{div}(u)$ is the classical normal stress tensor where $\lambda$ is the volumetric viscosity.

Remark 2. Let us remark that, if we play with the magnitude of viscosity $\mu_{i}$, the matrix $\Sigma$ will be useful to set a privileged flow direction.

The Navier-Stokes system is closed with the following boundary conditions on $\partial \Omega$ :

$$
\begin{aligned}
& \text { periodic conditions on } \partial \Omega_{x}, \\
& v_{\mid y=0}=v_{\mid y=H}=0, \\
& \partial_{y} \mathbf{u}_{\mid y=0}=\partial_{y} \mathbf{u}_{\mid y=H}=0 .
\end{aligned}
$$

We also assume that the distribution of the horizontal component of the velocity $\mathbf{u}$ and the density distribution are known at the initial time $t=0$ :

$$
\begin{aligned}
\mathbf{u}(0, x, y) & =\mathbf{u}_{0}(x, y), \\
\rho(0, x, y) & =\xi_{0}(x) e^{-g / c^{2} y}
\end{aligned}
$$

where $\xi_{0}$ is a bounded positive function:

$$
0 \leqslant \xi_{0}(x) \leqslant M<+\infty .
$$

Remark 3. The expression of $\rho$ at time $t=0$ is quite natural since in the atmosphere the density is stratified, i.e. for each altitude $y$, the density has the profile of the given function $\xi_{0}$. Moreover, it is also mathematically justified at the end of Section 2.1, more precisely see Equation (13).

\subsection{Formal derivation of the CPEs}

Taking advantages of the shallowness of the atmosphere, we assume that the characteristic scale for the altitude $H$ is small with respect to the characteristic length $L$. In this context, we also assume that the vertical movements and variations are very small compared to the horizontal ones which justifies the following approximation: let $\varepsilon$ be a "small" parameter such as:

$$
\varepsilon=\frac{H}{L}=\frac{V}{U}
$$

where $V$ and $U$ are respectively the characteristic scale of the vertical and horizontal velocity. We introduce the characteristic time $T$ such as: $T=\frac{L}{U}$ and 
the pressure unit $P=\bar{\rho} U^{2}$ where $\bar{\rho}$ is a characteristic density. Finally, we note the dimensionless quantities of time, space, fluid velocity, pressure, density and viscosities:

$$
\begin{gathered}
\tilde{t}=\frac{t}{T}, \quad \widetilde{x}=\frac{x}{L}, \quad \widetilde{y}=\frac{y}{H}, \quad \widetilde{u}=\frac{\mathbf{u}}{U}, \quad \widetilde{v}=\frac{v}{V}, \\
\tilde{p}=\frac{p}{\bar{\rho} U^{2}}, \quad \widetilde{\rho}=\frac{\rho}{\bar{\rho}}, \quad \widetilde{\lambda}=\frac{\lambda}{\bar{\lambda}}, \quad \widetilde{\mu}_{j}=\frac{\mu_{j}}{\bar{\mu}_{j}}, \quad j=1,2,3 .
\end{gathered}
$$

With these notations, the Froude number $F_{r}$, the Reynolds number associated to the viscosity $\mu_{i}, R e_{i},(i=1,2,3)$, the Reynolds number associated to the viscosity $\lambda, R e_{\lambda}$, and the Mach number $M_{a}$ are respectively:

$$
F_{r}=\frac{U}{\sqrt{g H}}, \quad R e_{i}=\frac{\bar{\rho} U L}{\overline{\mu_{i}}}, \quad R e_{\lambda}=\frac{\bar{\rho} U L}{\bar{\lambda}}, \quad M_{a}=\frac{U}{c} .
$$

Applying this scaling to System (4)-(7), using the definition of the dimensionless number (10) and dropping " ?" we get the following non-dimensional System:

$$
\left\{\begin{array}{r}
\partial_{t} \rho+\operatorname{div}_{x}(\rho \mathbf{u})+\partial_{y}(\rho v)=0 \\
\partial_{t}(\rho \mathbf{u})+\operatorname{div}_{x}(\rho \mathbf{u} \otimes \mathbf{u})+\partial_{y}(\rho v \mathbf{u})+\frac{1}{M_{a}^{2}} \nabla_{x} \rho+r \rho|\mathbf{u}| \mathbf{u}= \\
\frac{2}{R e_{1}} \operatorname{div}_{x}\left(\mu_{1} D_{x}(\mathbf{u})\right)+\frac{1}{R e_{2}} \partial_{y}\left(\mu_{2}\left(\frac{1}{\varepsilon^{2}} \partial_{y} \mathbf{u}+\nabla_{x} v\right)\right) \\
+\frac{1}{R e_{\lambda}} \nabla_{x}\left(\lambda \operatorname{div}_{x}(\mathbf{u})+\lambda \partial_{y} v\right) \\
\partial_{t}(\rho v)+\operatorname{div}_{x}(\rho \mathbf{u} v)+\partial_{y}\left(\rho v^{2}\right)+\frac{1}{\varepsilon^{2}} \frac{1}{M_{a}^{2}} \partial_{y} \rho=-\frac{1}{\varepsilon^{2}} \frac{1}{F_{r}^{2}} \rho \\
+\frac{1}{R e_{3}} \operatorname{div}_{x}\left(\mu_{3}\left(\frac{1}{\varepsilon^{2}} \partial_{y} \mathbf{u}+\nabla_{x} v\right)\right)+\frac{2}{\varepsilon^{2} R e_{3}} \partial_{y}\left(\mu_{3} \partial_{y} v\right) \\
+\frac{1}{\varepsilon^{2} R e_{\lambda}} \partial_{y}\left(\lambda \operatorname{div}_{x}(\mathbf{u})+\lambda \partial_{y} v\right)
\end{array}\right.
$$

where we have noted $R=\frac{r}{L}$.

Next, assuming the following asymptotic regime:

$$
\frac{\mu_{1}}{R e_{1}}=\nu_{1}, \frac{\mu_{i}}{R e_{i}}=\varepsilon^{2} \nu_{i}, i=2,3 \text { and } \frac{\lambda}{R e_{\lambda}}=\varepsilon^{2} \gamma
$$

and dropping all terms of order $O(\varepsilon)$, System (11) reduces to the following Compressible Primitive Equations (CPEs):

$$
\left\{\begin{array}{l}
\partial_{t} \rho+\operatorname{div}_{x}(\rho \mathbf{u})+\partial_{y}(\rho v)=0 \\
\partial_{t}(\rho \mathbf{u})+\operatorname{div}_{x}(\rho \mathbf{u} \otimes \mathbf{u})+\partial_{y}(\rho v \mathbf{u})+\frac{1}{M_{a}^{2}} \nabla_{x} \rho+r \rho|\mathbf{u}| \mathbf{u}= \\
2 \operatorname{div}_{x}\left(\nu_{1} D_{x}(\mathbf{u})\right)+\partial_{y}\left(\nu_{2} \partial_{y} \mathbf{u}\right) \\
\partial_{y} \rho=-\frac{M_{a}^{2}}{F_{r}^{2}} \rho
\end{array}\right.
$$


holding in the domain $\Omega=\left\{(x, y) ; x \in \Omega_{x} \subset \mathbb{R}^{2}, 0<y<1\right\}$.

Simplifying by setting $M_{a}=F_{r}$, the hydrostatic equation of System (12) gives:

$$
\rho(t, x, y)=\xi(t, x) e^{-y}
$$

for some function $\xi=\xi(t, x)$ that we call again "density".

Remark 4. This expression of the density justifies the choice of the initial data (9) for the density $\rho$.

In what follows, we note:

$$
\nu_{1}(t, x, y)=\bar{\nu}_{1} \rho(t, x, y) \text { and } \nu_{2}=\bar{\nu}_{2} \rho(t, x, y) e^{2 y} .
$$

for some positive constant $\bar{\nu}_{1}$ and $\bar{\nu}_{2}$.

\subsection{The main result}

In order to define a weak solution of the CPEs, we introduce the set of function $\rho \in \mathcal{P} \mathcal{E}\left(\mathbf{u}, v ; y, \rho_{0}\right)$ which satisfy

$$
\begin{array}{ll}
\rho \in L^{\infty}\left(0, T ; L^{3}(\Omega)\right), & \sqrt{\rho} \in L^{\infty}\left(0, T ; H^{1}(\Omega)\right), \\
\sqrt{\rho} \mathbf{u} \in L^{2}\left(0, T ;\left(L^{2}(\Omega)\right)^{2}\right), & \sqrt{\rho} v \in L^{\infty}\left(0, T ; L^{2}(\Omega)\right), \\
\sqrt{\rho} D_{x}(\mathbf{u}) \in L^{2}\left(0, T ;\left(L^{2}(\Omega)\right)^{2 \times 2}\right), & \sqrt{\rho} \partial_{y} v \in L^{2}\left(0, T ; L^{2}(\Omega)\right), \\
\nabla \sqrt{\rho} \in L^{2}\left(0, T ;\left(L^{2}(\Omega)\right)^{3}\right) &
\end{array}
$$

with $\rho \geqslant 0$ and where $(\rho, \sqrt{\rho} \mathbf{u}, \sqrt{\rho} v)$ satisfies:

$$
\left\{\begin{array}{l}
\partial_{t} \rho+\operatorname{div}_{x}(\sqrt{\rho} \sqrt{\rho} \mathbf{u})+\partial_{y}(\sqrt{\rho} \sqrt{\rho} v)=0, \\
\rho_{t=0}=\rho_{0} .
\end{array}\right.
$$

We also define the following integral operators for any smooth test function $\varphi$ with compact support such as $\varphi(T, x, y)=0$ and $\varphi_{0}=\varphi_{t=0}$ :

$$
\begin{aligned}
& \mathcal{A}(\rho, \mathbf{u}, v ; \varphi, d y)=-\int_{0}^{T} \int_{\Omega} \rho \mathbf{u} \partial_{t} \varphi d x d y d t \\
&+\int_{0}^{T} \int_{\Omega}\left(2 \nu_{1}(t, x, y) \rho D_{x}(\mathbf{u})-\rho \mathbf{u} \otimes \mathbf{u}\right): \nabla_{x} \varphi d x d y d t \\
&+\int_{0}^{T} \int_{\Omega} r \rho|\mathbf{u}| \mathbf{u} \varphi d x d y d t-\int_{0}^{T} \int_{\Omega} \rho \operatorname{div}(\varphi) d x d y d t \\
&-\int_{0}^{T} \int_{\Omega} \mathbf{u} \partial_{y}\left(\nu_{2}(t, x, y) \partial_{y} \varphi\right) d x d y d t \\
&-\int_{0}^{T} \int_{\Omega}^{\rho v \mathbf{u} \partial_{y} \varphi d x d y d t} \\
& \mathcal{B}(\rho, \mathbf{u}, v ; \varphi, d y)=\int_{0}^{T} \int_{\Omega} \rho v \varphi d x d y d t
\end{aligned}
$$

and

$$
\mathcal{C}(\rho, \mathbf{u} ; \varphi, d y)=\int_{\Omega} \rho_{\mid t=0} \mathbf{u}_{\mid t=0} \varphi_{0} d x d y
$$

Under these definitions, we consider weak solutions of the CPEs in sens of the distributions. More precisely, we will say that: 
Definition 1. A weak solution of System (12) on $[0, T] \times \Omega$, with boundary conditions (8) and initial conditions (9), is a collection of functions $(\rho, \boldsymbol{u}, v)$ such as $\rho \in \mathcal{P E}\left(\boldsymbol{u}, v ; y, \rho_{0}\right)$ and the following equality holds for all smooth test function $\varphi$ with compact support such as $\varphi(T, x, y)=0$ and $\varphi_{0}=\varphi_{t=0}$ :

$$
\mathcal{A}(\rho, \boldsymbol{u}, v ; \varphi, d y)+\mathcal{B}(\rho, \boldsymbol{u}, v ; \varphi, d y)=\mathcal{C}(\rho, \boldsymbol{u} ; \varphi, d y) .
$$

Then, we can state the main result:

Theorem 1. Let $\left(\rho_{n}, \boldsymbol{u}_{n}, v_{n}\right)$ be a sequence of weak solutions of System (12), with boundary conditions (8) and initial conditions (9), satisfying entropy inequalities (23) and (40) such as

$$
\rho_{n} \geqslant 0, \quad \rho_{0}^{n} \rightarrow \rho_{0} \text { in } L^{1}(\Omega), \quad \rho_{0}^{n} \boldsymbol{u}_{0}^{n} \rightarrow \rho_{0} \boldsymbol{u}_{0} \text { in } L^{1}(\Omega) .
$$

Then, up to a subsequence,

- $\rho_{n}$ converges strongly in $\mathcal{C}^{0}\left(0, T ; L^{3 / 2}(\Omega)\right)$,

- $\sqrt{\rho_{n}} \boldsymbol{u}_{n}$ converges strongly in $L^{2}\left(0, T ;\left(L^{3 / 2}(\Omega)\right)^{2}\right)$,

- $\rho_{n} u_{n}$ converges strongly in $L^{1}\left(0, T ;\left(L^{1}(\Omega)\right)^{2}\right)$ for all $T>0$,

- $\left(\rho_{n}, \sqrt{\rho_{n}} \boldsymbol{u}_{n}, \sqrt{\rho_{n}} v_{n}\right)$ converges to a weak solution of System (12),

- $\left(\rho_{n}, \boldsymbol{u}_{n}, v_{n}\right)$ satisfies the energy inequality (23), the entropy inequality (40) and converges to a weak solution of (12)-(8).

The proof of the main result is divided into three parts:

- in Sections 3.1, we perform a change of variables using $\left(\xi, \mathbf{u}, w=e^{-y} v\right)$ as unknowns instead of $(\rho, \mathbf{u}, v)$ and we obtain an intermediate model,

- in Section 3.2.2-3.2.6, we prove the stability of weak solutions of the model problem,

- in Section 3.2.7, by the reverse change of variables, we prove the main result.

\section{Stability of weak solutions for the CPEs}

As pointed out in Section 1, the classical techniques fails. To overpass this difficulty, following Ersoy et al. we perform a useful change of variables which transform the initial problem into a more simpler and more practical for mathematical analysis. 


\subsection{A model problem; an intermediate model}

Let us first remark that the structure of the density $\rho$, defined as a tensorial product (see Equation (13)), suggests the following change of variables:

$$
z=1-e^{-y}
$$

where the vertical velocity in the new coordinates becomes:

$$
w(t, x, z)=e^{-y} v(t, x, y) .
$$

Since the new vertical coordinate $z$ is defined as $\frac{d}{d y} z=e^{-y}$, multiplying by $e^{y}$ system (12) and using the viscosity profile (14) and the change of variables (17)-(18) provides the following model, called model problem:

$$
\left\{\begin{array}{l}
\partial_{t} \xi+\operatorname{div}_{x}(\xi \mathbf{u})+\partial_{z}(\xi w)=0, \\
\partial_{t}(\xi \mathbf{u})+\operatorname{div}_{x}(\xi \mathbf{u} \otimes \mathbf{u})+\partial_{z}(\xi \mathbf{u} w)+\nabla_{x} \xi+r \xi|\mathbf{u}| \mathbf{u}= \\
\partial_{z} \xi=0 \\
2 \bar{\nu}_{1} \operatorname{div}_{x}\left(\xi D_{x}(\mathbf{u})\right)+\bar{\nu}_{2} \partial_{z}\left(\xi \partial_{z} \mathbf{u}\right),
\end{array}\right.
$$

holding in the domain is $\Omega^{\prime}=\left\{(x, z) ; x \in \Omega_{x}^{\prime}, 0<z<1-e^{-1}\right\}$ where $\Omega_{x}^{\prime}=\mathbb{T}^{2}$ is the bi-dimensional torus.

In the new variables, the boundary conditions (8) and the initial conditions (9) become:

$$
\begin{aligned}
& \text { periodic conditions on } \Omega_{x}^{\prime}, \\
& w_{\mid z=0}=w_{\mid z=h}=0 \\
& \partial_{z} \mathbf{u}_{\mid z=0}=\partial_{z} \mathbf{u}_{\mid z=h}=0
\end{aligned}
$$

and

$$
\begin{aligned}
& \mathbf{u}(0, x, y)=\mathbf{u}_{0}(x, z) \\
& \xi(0, x)=\xi_{0}(x)
\end{aligned}
$$

where $h=1-e^{-1}$.

\subsection{Mathematical study of the model problem}

In this section, we show the stability of weak solutions of System (19). To this end, we will say that:

Definition 2. A weak solution of System (19) on $[0, T] \times \Omega^{\prime}$, with boundary (20) and initial conditions (21), is a collection of functions $(\xi, \boldsymbol{u}, w)$, if $\xi \in$ $\mathcal{P E}\left(\boldsymbol{u}, w ; z, \xi_{0}\right)$ and the following equality holds for all smooth test function $\varphi$ with compact support such as $\varphi(T, x, y)=0$ and $\varphi_{0}=\varphi_{t=0}$ :

$$
\mathcal{A}(\xi, \boldsymbol{u}, w ; \varphi, d z)=\mathcal{C}(\xi, \boldsymbol{u} ; \varphi, d z)
$$

where $\mathcal{A}$ and $\mathcal{C}$ are given by (15) and (16).

We then have the following result: 
Theorem 2. Let $\left(\xi_{n}, \boldsymbol{u}_{n}, w_{n}\right)$ be a sequence of weak solutions of System (19), with boundary conditions (20) and initial conditions (21), satisfying entropy inequalities (23) and (40) such as

$$
\xi_{n} \geqslant 0, \quad \xi_{0}^{n} \rightarrow \xi_{0} \text { in } L^{1}\left(\Omega^{\prime}\right), \quad \xi_{0}^{n} \boldsymbol{u}_{0}^{n} \rightarrow \xi_{0} \boldsymbol{u}_{0} \text { in } L^{1}\left(\Omega^{\prime}\right) .
$$

Then, up to a subsequence,

- $\xi_{n}$ converges strongly in $\mathcal{C}^{0}\left(0, T ; L^{3 / 2}\left(\Omega^{\prime}\right)\right)$,

- $\sqrt{\xi_{n}} \boldsymbol{u}_{n}$ converges strongly in $L^{2}\left(0, T ;\left(L^{3 / 2}\left(\Omega^{\prime}\right)\right)^{2}\right)$,

- $\xi_{n} u_{n}$ converges strongly in $L^{1}\left(0, T ;\left(L^{1}\left(\Omega^{\prime}\right)\right)^{2}\right)$ for all $T>0$,

- $\left(\xi_{n}, \sqrt{\xi_{n}} \boldsymbol{u}_{n}, \sqrt{\xi_{n}} w_{n}\right)$ converges to a weak solution of System (19),

- $\left(\xi_{n}, \boldsymbol{u}_{n}, w_{n}\right)$ satisfies the energy inequality (23), the entropy inequality (40) and converges to a weak solution of (19)-(20).

We divide the proof of Theorem 2 into three steps:

- in Section 3.2.1, we obtain suitable a priori bounds on $(\xi, \mathbf{u}, w)$,

- in Sections 3.2.2-3.2.5, we show the compactness of sequences $\left(\xi_{n}, \mathbf{u}_{n}, w_{n}\right)$ in apropriate space function,

- in Section 3.2.6, we prove that we can pass to the limit in all terms of System (19) which ends the proof of Theorem 2.

\subsubsection{Energy and entropy estimates}

A part of a priori bounds on $(\xi, \mathbf{u}, w)$ are obtained by the physical energy inequality which is obtained in a classical way by multiplying the momentum equation by $\mathbf{u}$, using the mass equation and integrating by parts. We obtain the following inequality:

$$
\begin{array}{r}
\frac{d}{d t} \int_{\Omega^{\prime}}\left(\xi \frac{\mathbf{u}^{2}}{2}+(\xi \ln \xi-\xi+1)\right) d x d z+\int_{\Omega^{\prime}} \xi\left(2 \bar{\nu}_{1}\left|D_{x}(\mathbf{u})\right|^{2}+\bar{\nu}_{2}\left|\partial_{z} \mathbf{u}\right|^{2}\right) d x d z \\
+r \int_{\Omega^{\prime}} \xi|\mathbf{u}|^{3} d x d z \leqslant 0
\end{array}
$$

which provides the uniform estimates:

$\sqrt{\xi} \mathbf{u}$ is bounded in $L^{\infty}\left(0, T ;\left(L^{2}\left(\Omega^{\prime}\right)\right)^{2}\right)$,

$\xi^{1 / 3} \mathbf{u}$ is bounded in $L^{3}\left(0, T ;\left(L^{3}\left(\Omega^{\prime}\right)\right)^{2}\right)$,

$\sqrt{\xi} \partial_{z} \mathbf{u}$ is bounded in $L^{2}\left(0, T ;\left(L^{2}\left(\Omega^{\prime}\right)\right)^{2}\right)$,

$\sqrt{\xi} D_{x}(\mathbf{u})$ is bounded in $L^{2}\left(0, T ;\left(L^{2}\left(\Omega^{\prime}\right)\right)^{2 \times 2}\right)$,

$\xi \ln \xi-\xi+1$ is bounded in $L^{\infty}\left(0, T ; L^{1}\left(\Omega^{\prime}\right)\right)$. 
The strong convergence of $\sqrt{\xi} \mathbf{u}$ required to pass to the limit in the non linear term $\xi \mathbf{u} \otimes \mathbf{u}$ is obtained by the mathematical BD-entropy. To this end, we first take the gradient of the mass equation, then we multiply by $2 \bar{\nu}_{1}$ and write the term $\nabla_{x} \xi$ as $\xi \nabla_{x} \ln \xi$ to obtain:

$$
\begin{array}{r}
\partial_{t}\left(2 \bar{\nu}_{1} \xi \nabla_{x} \ln \xi\right)+\operatorname{div}_{x}\left(2 \bar{\nu}_{1} \xi \nabla_{x} \ln \xi \otimes \mathbf{u}\right)+\partial_{z}\left(2 \bar{\nu}_{1} \xi \nabla_{x} \ln \xi w\right)+\operatorname{div}_{x}\left(2 \bar{\nu}_{1} \xi \nabla_{x}^{t} \mathbf{u}\right) \\
+\partial_{z}\left(2 \bar{\nu}_{1} \xi \nabla_{x} w\right)=0
\end{array}
$$

Next, we sum Equation (29) with the momentum equation of System (19) to get the equation:

$$
\begin{array}{r}
\partial_{t}\left(\xi\left(\mathbf{u}+2 \bar{\nu}_{1} \nabla_{x} \ln \xi\right)\right)+\operatorname{div}_{x}\left(\xi\left(\mathbf{u}+2 \bar{\nu}_{1} \nabla_{x} \ln \xi\right) \otimes \mathbf{u}\right)+\partial_{z}(\xi w \mathbf{u})+2 \bar{\nu}_{1} \partial_{z} \nabla(\xi w) \\
-2 \bar{\nu}_{1} \operatorname{div}_{x}\left(\xi A_{x}(\mathbf{u})\right)-\bar{\nu}_{2} \partial_{z}\left(\xi \partial_{z} \mathbf{u}\right)+r \xi|\mathbf{u}| \mathbf{u}+\nabla_{x} \xi=0,
\end{array}
$$

where $A_{x}(\mathbf{u})=\frac{\nabla_{x} \mathbf{u}-\nabla_{x}^{t} \mathbf{u}}{2}$ is the vorticity tensor. The mathematical BDentropy inequality is then obtained by multiplying the previous equation by $\mathbf{u}+2 \bar{\nu}_{1} \nabla_{x} \ln \xi$ and by integrating by parts. To this end, multiplying Equation (30) by the term $\mathbf{u}+2 \bar{\nu}_{1} \nabla_{x} \ln \xi$ and integrating over $\Omega^{\prime}$, we have to compute each term of the following integral:

$$
\begin{aligned}
& \int_{\Omega^{\prime}} \partial_{t}\left(\xi\left(\mathbf{u}+2 \bar{\nu}_{1} \nabla_{x} \ln \xi\right)\right)\left(\mathbf{u}+2 \bar{\nu}_{1} \nabla_{x} \ln \xi\right) d x d z \\
& \quad+\int_{\Omega^{\prime}} \operatorname{div}_{x}\left(\xi\left(\mathbf{u}+2 \bar{\nu}_{1} \nabla_{x} \ln \xi\right) \otimes \mathbf{u}\right)\left(\mathbf{u}+2 \bar{\nu}_{1} \nabla_{x} \ln \xi\right) d x d z \\
& \quad+\int_{\Omega^{\prime}} \partial_{z}(\xi w \mathbf{u})\left(\mathbf{u}+2 \bar{\nu}_{1} \nabla_{x} \ln \xi\right)+2 \bar{\nu}_{1} \int_{\Omega^{\prime}} \partial_{z} \nabla(\xi w)\left(\mathbf{u}+2 \bar{\nu}_{1} \nabla_{x} \ln \xi\right) d x d z \\
& -2 \bar{\nu}_{1} \int_{\Omega^{\prime}} \operatorname{div}_{x}\left(\xi A_{x}(\mathbf{u})\right)\left(\mathbf{u}+2 \bar{\nu}_{1} \nabla_{x} \ln \xi\right) d x d z+r \int_{\Omega^{\prime}} \xi|\mathbf{u}| \mathbf{u}\left(\mathbf{u}+2 \bar{\nu}_{1} \nabla_{x} \ln \xi\right) d x d z \\
& -\bar{\nu}_{2} \int_{\Omega^{\prime}} \partial_{z}\left(\xi \partial_{z} \mathbf{u}\right)\left(\mathbf{u}+2 \bar{\nu}_{1} \nabla_{x} \ln \xi\right) d x d z+\int_{\Omega^{\prime}} \nabla_{x} \xi\left(\mathbf{u}+2 \bar{\nu}_{1} \nabla_{x} \ln \xi\right) d x d z=0 .
\end{aligned}
$$

The two first one reads as follows:

$$
\begin{aligned}
& \int_{\Omega^{\prime}} \partial_{t}\left(\xi\left(\mathbf{u}+2 \bar{\nu}_{1} \nabla_{x} \ln \xi\right)\right)\left(\mathbf{u}+2 \bar{\nu}_{1} \nabla_{x} \ln \xi\right) d x d z \\
& \quad+\int_{\Omega^{\prime}} \operatorname{div}_{x}\left(\xi\left(\mathbf{u}+\bar{\nu}_{1} \nabla_{x} \ln \xi\right) \otimes \mathbf{u}\right)\left(\mathbf{u}+2 \bar{\nu}_{1} \nabla_{x} \ln \xi\right) d x d z \\
& \quad=\frac{1}{2} \int_{\Omega^{\prime}} \xi \partial_{t}\left|\mathbf{u}+2 \bar{\nu}_{1} \nabla_{x} \ln \xi\right|^{2} d x d z+\int_{\Omega^{\prime}}\left(\mathbf{u}+2 \bar{\nu}_{1} \nabla_{x} \ln \xi\right)^{2} \partial_{t} \xi d x d z \\
& +\frac{1}{2} \int_{\Omega^{\prime}}(\xi \mathbf{u} \cdot \nabla)\left|\mathbf{u}+2 \bar{\nu}_{1} \nabla_{x} \ln \xi\right|^{2} d x d z+\int_{\Omega^{\prime}}\left(\mathbf{u}+2 \bar{\nu}_{1} \nabla_{x} \ln \xi\right)^{2} \operatorname{div}_{x}(\xi \mathbf{u}) d x d z
\end{aligned}
$$


which is also:

$$
\begin{aligned}
& \int_{\Omega^{\prime}} \partial_{t}\left(\xi\left(u+2 \bar{\nu}_{1} \nabla_{x} \ln \xi\right)\right)\left(\mathbf{u}+2 \bar{\nu}_{1} \nabla_{x} \ln \xi\right) d x d z \\
& \quad+\int_{\Omega^{\prime}} \operatorname{div}_{x}\left(\xi\left(\mathbf{u}+\bar{\nu}_{1} \nabla_{x} \ln \xi\right) \otimes \mathbf{u}\right)\left(\mathbf{u}+2 \bar{\nu}_{1} \nabla_{x} \ln \xi\right) d x d z \\
&=\frac{1}{2} \int_{\Omega^{\prime}} \xi \partial_{t}\left|\mathbf{u}+2 \bar{\nu}_{1} \nabla_{x} \ln \xi\right|^{2} d x d z-\frac{1}{2} \int_{\Omega^{\prime}} \operatorname{div}(\xi \mathbf{u})\left|\mathbf{u}+2 \bar{\nu}_{1} \nabla_{x} \ln \xi\right|^{2} d x d z \\
&+\int_{\Omega^{\prime}}\left(\mathbf{u}+2 \bar{\nu}_{1} \nabla_{x} \ln \xi\right)^{2}\left(\partial_{t} \xi+\operatorname{div}_{x}(\xi \mathbf{u})\right) d x d z .
\end{aligned}
$$

Remarking that

$$
\partial_{z}(\xi w \mathbf{u})=\partial_{z}\left(\xi w\left(\mathbf{u}+2 \bar{\nu}_{1} \nabla_{x} \ln \xi\right)\right)-2 \bar{\nu}_{1} \partial_{z} w \nabla_{x} \xi,
$$

we have:

$$
\begin{gathered}
\int_{\Omega^{\prime}} \partial_{z}\left(\xi w\left(\mathbf{u}+2 \bar{\nu}_{1} \nabla_{x} \ln \xi\right)\right)(\xi w \mathbf{u})\left(\mathbf{u}+2 \bar{\nu}_{1} \nabla_{x} \ln \xi\right) d x d z \\
-2 \bar{\nu}_{1} \int_{\Omega^{\prime}} \nabla_{x} \xi \partial_{z} w\left(\mathbf{u}+2 \bar{\nu}_{1} \nabla_{x} \ln \xi\right) d x d z \\
=\frac{1}{2} \int_{\Omega^{\prime}} \xi w \partial_{z}\left|\mathbf{u}+2 \bar{\nu}_{1} \nabla_{x} \ln \xi\right|^{2} d x d z+\int_{\Omega^{\prime}}\left(\mathbf{u}+2 \bar{\nu}_{1} \nabla_{x} \ln \xi\right)^{2} \partial_{z}(\xi w) d x d z+ \\
2 \bar{\nu}_{1} \int_{\Omega^{\prime}} w \nabla_{x} \xi \partial_{z} \mathbf{u} d x d z
\end{gathered}
$$

which is finally:

$$
\begin{aligned}
& \int_{\Omega^{\prime}} \xi w \mathbf{u} \partial_{z}(\xi w(\mathbf{u}+\left.\left.2 \bar{\nu}_{1} \nabla_{x} \ln \xi\right)\right)\left(u+2 \bar{\nu}_{1} \nabla_{x} \ln \xi\right) d x d z \\
&-2 \bar{\nu}_{1} \int_{\Omega^{\prime}} \nabla \xi \partial_{z} w\left(\mathbf{u}+2 \bar{\nu}_{1} \nabla_{x} \ln \xi\right) d x d z \\
&=-\frac{1}{2} \int_{\Omega^{\prime}}\left|\mathbf{u}+2 \bar{\nu}_{1} \nabla_{x} \ln \xi\right|^{2} \partial_{z}(\xi w) d x d z+\int_{\Omega^{\prime}}\left(\mathbf{u}+2 \bar{\nu}_{1} \nabla_{x} \ln \xi\right)^{2} \partial_{z}(\xi w) d x d z \\
&+2 \bar{\nu}_{1} \int_{\Omega^{\prime}} w \nabla_{x} \xi \partial_{z} \mathbf{u} d x d z .
\end{aligned}
$$

Summing (32) and (33), we obtain:

$$
\begin{aligned}
& \int_{\Omega^{\prime}} \partial_{t}(\xi(\mathbf{u}\left.\left.+2 \bar{\nu}_{1} \nabla_{x} \ln \xi\right)\right)\left(\mathbf{u}+2 \bar{\nu}_{1} \nabla_{x} \ln \xi\right) d x d z \\
&+\int_{\Omega^{\prime}} \operatorname{div}_{x}\left(\xi\left(\mathbf{u}+\bar{\nu}_{1} \nabla_{x} \ln \xi\right) \otimes \mathbf{u}\right)\left(\mathbf{u}+2 \bar{\nu}_{1} \nabla_{x} \ln \xi\right) d x d z \\
& \quad+\int_{\Omega^{\prime}} \partial_{z}(\xi w \mathbf{u})\left(\mathbf{u}+2 \bar{\nu}_{1} \nabla_{x} \ln \xi\right) d x d z \\
&=\frac{1}{2} \frac{d}{d t} \int_{\Omega^{\prime}} \xi\left|\mathbf{u}+2 \bar{\nu}_{1} \nabla_{x} \ln \xi\right|^{2} d x d z+2 \bar{\nu}_{1} \int_{\Omega^{\prime}} w \nabla_{x} \xi \partial_{z} \mathbf{u} d x d z
\end{aligned}
$$


The fourth term in Equation (31), i.e. $2 \bar{\nu}_{1} \int_{\Omega^{\prime}} \partial_{z} \nabla(\xi w)\left(u+2 \bar{\nu}_{1} \nabla_{x} \ln \xi\right) d x d z$ gives:

$$
\begin{aligned}
\int_{\Omega^{\prime}} \partial_{z} \nabla_{x}(\xi w)\left(\mathbf{u}+2 \bar{\nu}_{1} \nabla_{x} \ln \xi\right) d x d z & =-\int_{\Omega^{\prime}} \nabla_{x}(\xi w) \partial_{z} \mathbf{u} d x d z \\
& =\int_{\Omega^{\prime}} \xi w \partial_{z} \operatorname{div}_{x}(\mathbf{u}) d x d z \\
& =\int_{\Omega^{\prime}}\left(w \partial_{z} \operatorname{div}_{x}(\xi \mathbf{u})-w \nabla_{x} \xi \partial_{z} \mathbf{u}\right) d x d z
\end{aligned}
$$

Differentiating the equation of the conservation of the mass with respect to $z$,

$$
\partial_{z} \operatorname{div}_{x}(\xi \mathbf{u})=-\xi \partial_{z}^{2} w
$$

we get:

$$
\begin{aligned}
2 \bar{\nu}_{1} \int_{\Omega^{\prime}} \partial_{z} \nabla_{x}(\xi w)(\mathbf{u} & \left.+2 \bar{\nu}_{1} \nabla_{x} \ln \xi\right) d x d z=-2 \bar{\nu}_{1} \int_{\Omega^{\prime}}\left(\xi w \partial_{z}^{2} w-w \nabla_{x} \xi \partial_{z} \mathbf{u}\right) d x d z \\
& \left.=2 \bar{\nu}_{1} \int_{\Omega^{\prime}} \xi\left|\partial_{z} w\right|^{2} d x d z-2 \bar{\nu}_{1} \int_{\Omega^{\prime}} w \nabla_{x} \xi \partial_{z} \mathbf{u}\right) d x d z
\end{aligned}
$$

In order to compute the term $-2 \bar{\nu}_{1} \int_{\Omega^{\prime}} \operatorname{div}_{x}\left(\xi A_{x}(\mathbf{u})\right)\left(\mathbf{u}+2 \bar{\nu}_{1} \nabla_{x} \ln \xi\right) d x d z$ in Equation (31), we have just to remark that thanks to periodic conditions, we have

$$
\int_{\Omega^{\prime}} \operatorname{div}_{x}\left(\xi A_{x}(u)\right) \nabla_{x} \ln \xi d x d z=0
$$

which leads to:

$$
-2 \bar{\nu}_{1} \int_{\Omega^{\prime}} \operatorname{div}_{x}\left(\xi A_{x}(\mathbf{u})\right)\left(\mathbf{u}+2 \bar{\nu}_{1} \nabla_{x} \ln \xi\right) d x d z=2 \bar{\nu}_{1} \int_{\Omega^{\prime}} \xi\left|A_{x}(\mathbf{u})\right|^{2} d x d z
$$

The fifth and sixthterm in Equation (31) simply read:

$$
r \int_{\Omega^{\prime}} \xi|\mathbf{u}| \mathbf{u}\left(\mathbf{u}+2 \bar{\nu}_{1} \nabla_{x} \ln \xi\right) d x d z=r \int_{\Omega^{\prime}} \xi|\mathbf{u}|^{3} d x d z+2 \bar{\nu}_{1} r \int_{\Omega^{\prime}}|\mathbf{u}| \mathbf{u} \nabla \xi d x d z
$$

and

$$
-\bar{\nu}_{2} \int_{\Omega^{\prime}} \partial_{z}\left(\xi \partial_{z} \mathbf{u}\right)\left(\mathbf{u}+2 \bar{\nu}_{1} \nabla_{x} \ln \xi\right) d x d z=\bar{\nu}_{2} \int_{\Omega^{\prime}} \xi\left|\partial_{z} \mathbf{u}\right|^{2} d x d z .
$$

The last term $\int_{\Omega^{\prime}} \nabla_{x} \xi\left(\mathbf{u}+2 \bar{\nu}_{1} \nabla_{x} \ln \xi\right) d x d z$ gives

$$
\begin{aligned}
\int_{\Omega^{\prime}} \nabla_{x} \xi\left(\mathbf{u}+2 \bar{\nu}_{1} \nabla_{x} \ln \xi\right) d x d z=\frac{d}{d t} \int_{\Omega^{\prime}}(\xi \log \xi & -\xi+1) d x d z \\
& +8 \bar{\nu}_{1} \int_{\Omega^{\prime}}\left|\nabla_{x} \sqrt{\xi}\right|^{2} d x d z
\end{aligned}
$$


Finally, summing the terms (34) to (39), we obtain the entropy inequality:

$$
\begin{aligned}
\frac{1}{2} \frac{d}{d t} \int_{\Omega^{\prime}}(\xi \mid \mathbf{u} & \left.+\left.2 \bar{\nu}_{1} \nabla_{x} \ln \xi\right|^{2}+2(\xi \log \xi-\xi+1)\right) d x d z \\
& +\int_{\Omega^{\prime}} 2 \bar{\nu}_{1} \xi\left|\partial_{z} w\right|^{2}+2 \bar{\nu}_{1} \xi\left|A_{x}(u)\right|^{2}+\bar{\nu}_{2} \xi\left|\partial_{z} \mathbf{u}\right|^{2} d x d z \\
& \quad+\int_{\Omega^{\prime}} r \xi|\mathbf{u}|^{3}+2 \bar{\nu}_{1} r|\mathbf{u}| \mathbf{u} \nabla_{x} \xi+8 \bar{\nu}_{1}\left|\nabla_{x} \sqrt{\xi}\right|^{2} d x d z=0 .
\end{aligned}
$$

which gives the following estimates:

$$
\begin{array}{r}
\nabla \sqrt{\xi} \text { is bounded in } L^{\infty}\left(0, T ;\left(L^{2}\left(\Omega^{\prime}\right)\right)^{3}\right), \\
\sqrt{\xi} \partial_{z} w \text { is bounded in } L^{2}\left(0, T ; L^{2}\left(\Omega^{\prime}\right)\right), \\
\sqrt{\xi} A_{x}(\mathbf{u}) \text { is bounded in } L^{2}\left(0, T ;\left(L^{2}\left(\Omega^{\prime}\right)\right)^{2 \times 2}\right) .
\end{array}
$$

This finishes the first step of the proof of Theorem 2 .

Remark 5. Estimate (41) is a straightforward consequence of estimates $\sqrt{\xi}\left(\boldsymbol{u}+2 \bar{\nu}_{1} \nabla_{x} \ln \xi\right) \in L^{\infty}\left(0, T,\left(L^{2}\left(\Omega^{\prime}\right)\right)^{2}\right)$ and $\sqrt{\xi} \boldsymbol{u} \in L^{\infty}\left(0, T,\left(L^{2}\left(\Omega^{\prime}\right)\right)^{2}\right)$ since

$$
\sqrt{\xi}\left(\boldsymbol{u}+2 \bar{\nu}_{1} \nabla_{x} \ln \xi\right)=\sqrt{\xi} \boldsymbol{u}+2 \bar{\nu}_{1} \frac{\nabla_{x} \xi}{\sqrt{\xi}} .
$$

To show the compactness of sequences $\left(\xi_{n}, \mathbf{u}_{n}, w_{n}\right)$ in apropriate space function we follow the work of Mellet et al. [10]. To this end, we divid this second step of the proof of Theorem 2 into 4 parts :

1. in Section 3.2.2, we show the convergence of the sequence $\sqrt{\xi_{n}}$,

2. in Section 3.2.3, we seek bounds of $\sqrt{\xi_{n}} \mathbf{u}_{n}$ and $\sqrt{\xi_{n}} w_{n}$,

3. in Section 3.2.4, we prove the convergence of $\xi_{n} \mathbf{u}_{n}$,

4. in Section 3.2.5, we prove the convergence of $\sqrt{\xi_{n}} \mathbf{u}_{n}$.

\subsubsection{Convergence of $\sqrt{\xi_{n}}$}

Let us first prove the following

Lemma 1. For every $\xi_{n}$ satisfying the mass equation of System (19), we have:

$$
\begin{gathered}
\sqrt{\xi_{n}} \text { is bounded in } L^{\infty}\left(0, T, H^{1}\left(\Omega^{\prime}\right)\right), \\
\partial_{t} \sqrt{\xi_{n}} \text { is bounded in } L^{2}\left(0, T, H^{-1}\left(\Omega^{\prime}\right)\right) .
\end{gathered}
$$

Then, up to a subsequence, the sequence $\xi_{n}$ converges almost everywhere and strongly in $L^{2}\left(0, T ; L^{2}\left(\Omega^{\prime}\right)\right)$. Moreover, $\xi_{n}$ converges to $\xi$ in $\mathcal{C}^{0}\left(0, T ; L^{3 / 2}\left(\Omega^{\prime}\right)\right)$.

\section{Proof of Lemma 1:}

$\sqrt{\xi_{n}}$ is bounded in $L^{\infty}\left(0, T, H^{1}\left(\Omega^{\prime}\right)\right)$ since we have

$$
\left\|\sqrt{\xi_{n}}(t)\right\|_{L^{2}\left(\Omega^{\prime}\right)}^{2}=\left\|\xi_{0}^{n}\right\|_{L^{1}\left(\Omega^{\prime}\right)}
$$


from the continuity equation and by Estimate (41).

Using again the mass conservation equation, we write:

$$
\begin{aligned}
\partial_{t}\left(\sqrt{\xi_{n}}\right) & =-\frac{1}{2} \sqrt{\xi_{n}} \operatorname{div}_{x}\left(\mathbf{u}_{n}\right)-\mathbf{u}_{n} \cdot \nabla_{x} \sqrt{\xi_{n}}-\sqrt{\xi_{n}} \partial_{z} w_{n} \\
& =\frac{1}{2} \sqrt{\xi_{n}} \operatorname{div}_{x}\left(\mathbf{u}_{n}\right)-\operatorname{div}_{x}\left(\mathbf{u}_{n} \sqrt{\xi_{n}}\right)-\sqrt{\xi_{n}} \partial_{z} w_{n} .
\end{aligned}
$$

Then, from Estimates (27), (41), (42) and (43), we get:

$$
\partial_{t} \sqrt{\xi_{n}} \text { is bounded in } L^{2}\left(0, T, H^{-1}\left(\Omega^{\prime}\right)\right) \text {. }
$$

We have then the compactness of $\sqrt{\xi_{n}}$ in $\mathcal{C}^{0}\left(0, T, L^{2}\left(\Omega^{\prime}\right)\right.$ by Aubin's Lemma, i.e.

$$
\sqrt{\xi_{n}} \text { converges strongly to } \sqrt{\xi} \text { in } \mathcal{C}^{0}\left(0, T, L^{2}\left(\Omega^{\prime}\right)\right) \text {. }
$$

We also have, by Sobolev embeddings, bounds of $\sqrt{\xi_{n}}$ in spaces $L^{\infty}\left(0, T, L^{p}\left(\Omega^{\prime}\right)\right)$ for all $p \in[1,6]$. Consequently, for $p=6$, we get bounds of

$$
\xi_{n} \text { in } L^{\infty}\left(0, T, L^{3}\left(\Omega^{\prime}\right)\right)
$$

and we deduce that:

$$
\xi_{n} \mathbf{u}_{n}=\sqrt{\xi_{n}} \sqrt{\xi_{n}} \mathbf{u}_{n} \text { is bounded in } L^{\infty}\left(0, T, L^{3 / 2}\left(\Omega^{\prime}\right)^{2}\right) .
$$

It follows that $\partial_{t} \xi_{n}$ is bounded in $L^{\infty}\left(0, T, W^{-1,3 / 2}\left(\Omega^{\prime}\right)\right)$ since

$$
\partial_{t} \xi_{n}=-\operatorname{div}_{x}\left(\xi_{n} \mathbf{u}_{n}\right)-\xi_{n} \partial_{z} w_{n}
$$

and Estimate (42) holds.

To conclude, writing

$$
\nabla_{x} \xi_{n}=2 \sqrt{\xi_{n}} \nabla_{x} \sqrt{\xi_{n}} \in L^{\infty}\left(0, T ; L^{3 / 2}\left(\Omega^{\prime}\right)^{2}\right),
$$

we deduce bounds of $\xi_{n}$ in $L^{\infty}\left(0, T ; W^{1,3 / 2}\left(\Omega^{\prime}\right)\right)$. Then, using again Aubin's lemma provides compactness of $\xi_{n}$ in the intermediate space $L^{3 / 2}\left(\Omega^{\prime}\right)$ :

$$
\text { compactness of } \xi_{n} \text { in } \mathcal{C}^{0}\left(0, T ; L^{3 / 2}\left(\Omega^{\prime}\right)\right) \text {. }
$$

3.2.3. Bounds of $\sqrt{\xi_{n}} \boldsymbol{u}_{n}$ and $\sqrt{\xi_{n}} w_{n}$

To prove the convergence of the momentum, we have to control bounds of $\sqrt{\xi_{n}} \mathbf{u}_{n}$ and $\sqrt{\xi_{n}} w_{n}$. Thus, we have to prove the following

Lemma 2. We have

$$
\sqrt{\xi_{n}} \boldsymbol{u}_{n} \text { bounded in } L^{\infty}\left(0, T ;\left(L^{2}\left(\Omega^{\prime}\right)\right)^{2}\right)
$$

and

$$
\sqrt{\xi} w_{n} \text { bounded in } L^{2}\left(0, T ; L^{2}\left(\Omega^{\prime}\right)\right) \text {. }
$$


Proof of Lemma 2: We have already bounds of $\sqrt{\xi_{n}}$ (see Estimates (24)). There is left to show bounds of $\sqrt{\xi_{n}} w_{n}$ in $L^{2}\left(0, T ; L^{2}\left(\Omega^{\prime}\right)\right)$. As $\xi_{n}=\xi_{n}(t, x)$ and Estimates (42) holds, by the Poincaré inequality, we have:

$$
\int_{0}^{h}\left|\sqrt{\xi_{n}} w_{n}\right|^{2} d z \leqslant c \int_{0}^{h}\left|\partial_{z}\left(\sqrt{\xi_{n}} w_{n}\right)\right|^{2} d z .
$$

Consequently, the following inequality

$$
\int_{\Omega^{\prime}} \xi_{n}\left|w_{n}\right|^{2} d x d z \leqslant c \int_{\Omega^{\prime}} \xi_{n}\left|\partial_{z} w_{n}\right|^{2} d x d z
$$

gives bounds of $\sqrt{\xi_{n}} w_{n}$ in $L^{2}\left(0, T ; L^{2}\left(\Omega^{\prime}\right)\right)$.

\subsubsection{Convergence of $\xi_{n} \boldsymbol{u}_{n}$}

As bounds of $\sqrt{\xi_{n}} \mathbf{u}_{n}$ and $\sqrt{\xi_{n}} w_{n}$ are provided by Lemma 2, we are able to show the convergence of the momentum.

Lemma 3. Let $m_{n}=\xi_{n} \boldsymbol{u}_{n}$ be a sequence satisfying the momentum equation (19). Then we have:

$$
\xi_{n} \boldsymbol{u}_{n} \rightarrow m \quad \text { in } L^{2}\left(0, T ;\left(L^{p}\left(\Omega^{\prime}\right)\right)^{2}\right) \text { strong }, \quad \forall 1 \leqslant p<3 / 2
$$

and

$$
\xi_{n} \boldsymbol{u}_{n} \rightarrow m \text { a.e. }(t, x, y) \in(0, T) \times \Omega^{\prime} .
$$

Proof of Lemma 3:

Writing $\nabla_{x}\left(\xi_{n} \mathbf{u}_{n}\right)$ as:

$$
\nabla_{x}\left(\xi_{n} \mathbf{u}_{n}\right)=\sqrt{\xi_{n}} \sqrt{\xi_{n}} \nabla_{x} \mathbf{u}_{n}+2 \sqrt{\xi_{n}} \mathbf{u}_{n} \otimes \nabla_{x} \sqrt{\xi_{n}}
$$

provides

$$
\nabla_{x}\left(\xi_{n} \mathbf{u}_{n}\right) \text { bounded in } L^{2}\left(0, T ;\left(L^{1}\left(\Omega^{\prime}\right)\right)^{2 \times 2}\right) .
$$

Next, we have

$$
\partial_{z}\left(\xi_{n} \mathbf{u}_{n}\right)=\sqrt{\xi_{n}} \sqrt{\xi_{n}} \partial_{z}\left(\mathbf{u}_{n}\right) \text { is bounded } L^{2}\left(0, T ;\left(L^{3 / 2}\left(\Omega^{\prime}\right)\right)^{2}\right) .
$$

Then, from bounds (44) and (45), we deduce:

$$
\xi_{n} \mathbf{u}_{n} \text { is bounded } L^{2}\left(0, T ;\left(W^{1,1}\left(\Omega^{\prime}\right)\right)^{2}\right) .
$$

On the other hand, we have:

$$
\begin{aligned}
\partial_{t}\left(\xi_{n} \mathbf{u}_{n}\right)= & -\operatorname{div}_{x}\left(\xi_{n} \mathbf{u}_{n} \otimes \mathbf{u}_{n}\right)-\partial_{z}\left(\xi_{n} \mathbf{u}_{n} w_{n}\right)-\nabla_{x} \xi_{n} \\
& +2 \bar{\nu}_{1} \operatorname{div}_{x}\left(\xi_{n} D_{x}\left(\mathbf{u}_{n}\right)\right)+\bar{\nu}_{2} \partial_{z}\left(\xi_{n} \partial_{z} \mathbf{u}_{n}\right) \\
& -r \xi_{n}\left|\mathbf{u}_{n}\right| \mathbf{u}_{n}
\end{aligned}
$$


As

$$
\xi_{n} \mathbf{u}_{n} \otimes \mathbf{u}_{n}=\sqrt{\xi} \mathbf{u}_{n} \otimes \sqrt{\xi} \mathbf{u}_{n},
$$

we deduce bounds of

$$
\xi_{n} \mathbf{u}_{n} \otimes \mathbf{u}_{n} \text { in } L^{\infty}\left(0, T ;\left(L^{1}\left(\Omega^{\prime}\right)\right)^{2 \times 2}\right) .
$$

Particularly, we have

$$
\operatorname{div}_{x}\left(\xi_{n} \mathbf{u}_{n} \otimes \mathbf{u}_{n}\right) \text { bounded in } L^{\infty}\left(0, T ;\left(W^{-2,4 / 3}\left(\Omega^{\prime}\right)\right)^{2}\right) .
$$

Similarly, as $\xi_{n} \mathbf{u}_{n} w_{n}=\sqrt{\xi} \mathbf{u}_{n} \sqrt{\xi} w_{n} \in\left(L^{1}\left(\Omega^{\prime}\right)\right)^{2}$, we also have:

$$
\partial_{z}\left(\xi_{n} \mathbf{u}_{n} w_{n}\right) \text { bounded in } L^{\infty}\left(0, T ;\left(W^{-2,4 / 3}\left(\Omega^{\prime}\right)\right)^{2}\right) .
$$

Moreover, as $\sqrt{\xi_{n}} \sqrt{\xi_{n}} \partial_{z} \mathbf{u}_{n} \in L^{2}\left(0, T ;\left(L^{3 / 2}\left(\Omega^{\prime}\right)\right)^{2}\right)$ and $\sqrt{\xi_{n}} \sqrt{\xi_{n}} D_{x}\left(\mathbf{u}_{n}\right) \in$ $L^{2}\left(0, T ;\left(L^{3 / 2}\left(\Omega^{\prime}\right)\right)^{2 \times 2}\right)$, we get bounds of

$$
\partial_{z}\left(\sqrt{\xi_{n}} \sqrt{\xi_{n}} \partial_{z} \mathbf{u}_{n}\right), \operatorname{div}_{x}\left(\sqrt{\xi_{n}} \sqrt{\xi_{n}} D_{x}\left(\mathbf{u}_{n}\right)\right) \in L^{2}\left(0, T ;\left(W^{-1,3 / 2}\left(\Omega^{\prime}\right)\right)^{2}\right) .
$$

We also have bounds of $\nabla_{x} \xi_{n} \in L^{\infty}\left(0, T,\left(W^{-1,3 / 2}\left(\Omega^{\prime}\right)\right)^{2}\right)$.

Using $W^{-1,3 / 2}\left(\Omega^{\prime}\right) \subset W^{-1,4 / 3}\left(\Omega^{\prime}\right)$, we obtain

$$
\partial_{t}\left(\xi_{n} \mathbf{u}_{n}\right) \text { bounded in } L^{2}\left(0, T ;\left(W^{-2,4 / 3}\left(\Omega^{\prime}\right)\right)^{2}\right) .
$$

Using Aubin's Lemma with the bounds (46), (48) provides the compactness of

$$
\xi_{n} \mathbf{u}_{n} \in L^{2}\left(0, T ;\left(L^{p}\left(\Omega^{\prime}\right)\right)^{2}\right), \forall p \in[1,3 / 2[.
$$

3.2.5. Convergence of $\sqrt{\xi_{n}} \boldsymbol{u}_{n}$ and $\xi_{n} w_{n}$

Let us note that, up to Section 3.2.4, we can always define $\mathbf{u}=m / \xi$ on the set $\{\xi>0\}$, but we do not know, a priori, if $m$ equals zero on the vacuum set. To this end, we need to prove the following lemma:

\section{Lemma 4.}

1. The sequence $\sqrt{\xi_{n}} \boldsymbol{u}_{n}$ satisfies

- $\sqrt{\xi_{n}} \boldsymbol{u}_{n}$ converges strongly in $L^{2}\left(0, T ;\left(L^{2}\left(\Omega^{\prime}\right)\right)^{2}\right)$ to $\frac{m}{\sqrt{\xi}}$.

- We have $m=0$ almost everywhere on the set $\{\xi=0\}$ and there exists a function $\boldsymbol{u}$ such that $m=\xi \boldsymbol{u}$ and

$$
\begin{aligned}
\xi_{n} \boldsymbol{u}_{n} \rightarrow & \xi \boldsymbol{u} \text { strongly in } L^{2}\left(0, T ;\left(L^{p}\left(\Omega^{\prime}\right)\right)^{2}\right) \text { for all } p \in[1,3 / 2[, \\
& \sqrt{\xi_{n}} \boldsymbol{u}_{n} \rightarrow \sqrt{\xi} \boldsymbol{u} \text { strongly in } L^{2}\left(0, T ;\left(L^{2}\left(\Omega^{\prime}\right)\right)^{2}\right) .
\end{aligned}
$$

2. The sequence $\sqrt{\xi_{n}} w_{n}$ converges weakly in $L^{2}\left(0, T ; L^{2}\left(\Omega^{\prime}\right)\right)$ to $\sqrt{\xi} w$. 
To prove Lemma 4, we adapt the proof of Mellet et al. [10]. As already pointed out by Bresch et al. [3], the presence of the term $r \xi|\mathbf{u}| \mathbf{u}$ simplify also this proof.

Proof of Lemma 4:

To start, we set $m_{n}=\xi_{n} \mathbf{u}_{n}$.

Since $\frac{m_{n}}{\sqrt{\xi_{n}}}$ is bounded in $L^{\infty}\left(0, T ;\left(L^{2}\left(\Omega^{\prime}\right)\right)^{2}\right)$ Fatou's lemma yields:

$$
\int_{\Omega^{\prime}} \liminf \frac{m_{n}^{2}}{\xi_{n}}<\infty
$$

In particular, we have $m(t, x, z)=0$ almost everywhere on the set $\{\xi(t, x)=0\}$. So, if we define the limit velocity $\mathbf{u}(t, x, z)$ by setting

$$
\mathbf{u}(t, x, z)=\left\{\begin{array}{lll}
\frac{m(t, x, z)}{\xi(t, x)} & \text { if } & \xi(t, x) \neq 0 \\
\mathbf{u}(t, x, z)=0 & \text { if } & \xi(t, x)=0
\end{array}\right.
$$

then, we have

$$
m(t, x, z)=\xi(t, x) \mathbf{u}(t, x, z)
$$

and

$$
\int_{\Omega^{\prime}} \frac{m^{2}}{\xi} d x d z=\int_{\Omega^{\prime}} \xi \mathbf{u}^{2} d x d z<\infty .
$$

Next, since $m_{n}$ and $\sqrt{\xi_{n}}$ converge almost everywhere, it is readily seen that on the set $\{\xi(t, x) \neq 0\}$,

$$
\sqrt{\xi_{n}} \mathbf{u}_{n}=\frac{m_{n}}{\sqrt{\xi_{n}}} \text { converges almost everywhere to } \sqrt{\xi} \mathbf{u}=\frac{m}{\sqrt{\xi}} .
$$

Moreover, for a constant $M>0$, we have:

$$
\sqrt{\xi_{n}} \mathbf{u}_{n} \mathbb{1}_{\left|\mathbf{u}_{n}\right| \leq M} \rightarrow \sqrt{\xi} \mathbf{u} \mathbb{1}_{|\mathbf{u}| \leq M} \text { almost everywhere. }
$$

As a matter of fact, the convergence holds almost everywhere on the set

$$
\{\xi(t, x) \neq 0\} \cup\{\xi(t, x)=0\}
$$

and we have

$$
\sqrt{\xi_{n}} \mathbf{u}_{n} \mathbb{1}_{\left|\mathbf{u}_{n}\right| \leq M} \leq M \sqrt{\xi} .
$$

To complete the proof, we cut the $L^{2}$ norm as follows

$$
\begin{aligned}
\int_{\Omega^{\prime}}\left|\sqrt{\xi_{n}} \mathbf{u}_{n}-\sqrt{\xi} \mathbf{u}\right|^{2} d x d z \leq & \int_{\Omega^{\prime}}\left|\sqrt{\xi_{n}} \mathbf{u}_{n} \mathbb{1}_{\left|\mathbf{u}_{n}\right| \leq M}-\sqrt{\xi} \mathbf{u} \mathbb{1}_{|\mathbf{u}| \leq M}\right|^{2} d x d z \\
& +2 \int_{\Omega^{\prime}}\left|\sqrt{\xi_{n}} \mathbf{u}_{n} \mathbb{1}_{\left|\mathbf{u}_{n}\right| \geq M}\right|^{2} d x d z \\
& +2 \int_{\Omega^{\prime}}\left|\sqrt{\xi} \mathbf{u} \mathbb{1}_{|\mathbf{u}| \geq M}\right|^{2} d x d z
\end{aligned}
$$


It is obvious that $\sqrt{\xi_{n}} \mathbf{u}_{n} \mathbb{1}_{\left|\mathbf{u}_{n}\right| \leq M}$ is uniformly bounded in $L^{\infty}\left(0, T ;\left(L^{2}\left(\Omega^{\prime}\right)\right)^{2}\right)$, then using (49) gives the convergence of the first integral:

$$
\int_{\Omega^{\prime}}\left|\sqrt{\xi_{n}} \mathbf{u}_{n} \mathbb{1}_{\left|\mathbf{u}_{n}\right| \leq M}-\sqrt{\xi} \mathbf{u} \mathbb{1}_{|\mathbf{u}| \leq M}\right|^{2} d x d z \rightarrow 0 .
$$

Finally, writing

$$
\begin{gathered}
\int_{\Omega^{\prime}}\left|\sqrt{\xi_{n}} \mathbf{u}_{n} \mathbb{1}_{\left|\mathbf{u}_{n}\right| \geq M}\right|^{2} d x d z \leq \frac{1}{M} \int_{\Omega^{\prime}} \xi_{n}\left|\mathbf{u}_{n}\right|^{3} d x d z \\
\int_{\Omega^{\prime}}\left|\sqrt{\xi} \mathbf{u} \mathbb{1}_{|\mathbf{u}| \geq M}\right|^{2} d x d z \leq \frac{1}{M} \int_{\Omega^{\prime}} \xi|\mathbf{u}|^{3} d x d z
\end{gathered}
$$

and putting together (50), (51) and (52), we deduce:

$$
\lim _{n \rightarrow+\infty} \sup \int_{\Omega^{\prime}}\left|\sqrt{\xi_{n}} \mathbf{u}_{n}-\sqrt{\xi} \mathbf{u}\right|^{2} d x d z \leq \frac{C}{M}, \forall M>0
$$

which ends the first point of the lemma by taking $M \rightarrow+\infty$.

The second part of the theorem is done by weak compactness. As $\sqrt{\xi_{n}} w_{n}$ is bounded in $L^{2}\left(0, T ; L^{2}\left(\Omega^{\prime}\right)\right)$, there exists, up to a subsequence, $\sqrt{\xi_{n}} w_{n}$ which converges weakly to some limit $l$ in $L^{2}\left(0, T ; L^{2}\left(\Omega^{\prime}\right)\right)$. Next, we define $w$ as:

$$
w=\left\{\begin{array}{lll}
\frac{l}{\sqrt{\xi}} & \text { if } & \xi>0 \\
0 \text { a.e. } & \text { if } & \xi=0
\end{array}\right.
$$

where the limit $l$ is written: $l=\sqrt{\xi} \frac{l}{\sqrt{\xi}}=\sqrt{\xi} w$.

This finishes the second point of the proof of Theorem 2 .

\subsubsection{Convergence step}

Gathering the previous results, we show straightforwardly that we can pass to the limit in all terms of System (19) in the sense of Theorem 2. To this end, let $\left(\xi_{n}, \mathbf{u}_{n}, w_{n}\right)$ be a weak solution of System (19) satisfying Lemma 1 to 4 and let $\phi \in \mathcal{C}_{c}^{\infty}\left([0, T] \times \Omega^{\prime}\right)$ be a smooth function with compact support such as $\phi(T, x, z)=0$ and $\phi(0, x, z)=\phi_{0}(x, z)$. Then, writing each term of the weak formulation of System (19), we have:

- For the first integral, we have:

$$
\begin{aligned}
\int_{0}^{T} \int_{\Omega^{\prime}} \partial_{t}\left(\xi_{n} \mathbf{u}_{n}\right) \phi d x d z d t= & -\int_{0}^{T} \int_{\Omega^{\prime}} \xi_{n} \mathbf{u}_{n} \partial_{t} \phi d x d z d t \\
& -\int_{\Omega^{\prime}}^{\xi_{0}^{n} \mathbf{u}_{0}^{n} \phi_{0} d x d z}
\end{aligned}
$$


Using convergences (22) and Lemma 3, we get

$$
\begin{gathered}
-\int_{0}^{T} \int_{\Omega^{\prime}} \xi_{n} \mathbf{u}_{n} \partial_{t} \phi d x d z d t-\int_{\Omega^{\prime}} \xi_{0}^{n} \mathbf{u}_{0}^{n} \phi(0, x, z) d x d z \rightarrow \\
-\int_{0}^{T} \int_{\Omega^{\prime}} \xi \mathbf{u} \partial_{t} \phi d x d z d t-\int_{\Omega^{\prime}} \xi_{0} \mathbf{u}_{0} \phi(0, x, y) d x d z .
\end{gathered}
$$

- For the following integral, we write:

$\int_{0}^{T} \int_{\Omega^{\prime}} \operatorname{div}_{x}\left(\xi_{n} \mathbf{u}_{n} \otimes \mathbf{u}_{n}\right) \cdot \phi d x d z d t=-\int_{0}^{T} \int_{\Omega^{\prime}} \xi_{n} \mathbf{u}_{n} \otimes \mathbf{u}_{n}: \nabla_{x} \phi d x d z d t$ then, from Equality (47) and Lemma 4, we have:

$$
-\int_{0}^{T} \int_{\Omega^{\prime}} \xi_{n} \mathbf{u}_{n} \otimes \mathbf{u}_{n}: \nabla_{x} \phi d x d z d t \rightarrow-\int_{0}^{T} \int_{\Omega^{\prime}} \xi \mathbf{u} \otimes \mathbf{u}: \nabla_{x} \phi d x d z d t .
$$

- Writing

$$
\int_{0}^{T} \int_{\Omega^{\prime}} \partial_{z}\left(\xi_{n} \mathbf{u}_{n} w_{n}\right) \cdot \phi d x d z d t=-\int_{0}^{T} \int_{\Omega^{\prime}} \xi_{n} \mathbf{u}_{n} w_{n} \cdot \partial_{z} \phi d x d z d t,
$$

as $\xi_{n} \mathbf{u}_{n} w_{n}=\sqrt{\xi_{n}} \mathbf{u}_{n} \sqrt{\xi_{n}} w_{n}$, by Lemma 4 , we get:

$$
-\int_{0}^{T} \int_{\Omega^{\prime}} \xi_{n} \mathbf{u}_{n} w_{n} \cdot \partial_{z} \phi d x d z d t \rightarrow-\int_{0}^{T} \int_{\Omega^{\prime}} \xi \mathbf{u} w \cdot \partial_{z} \phi d x d z d t .
$$

- For the following integral, we write:

$$
\int_{0}^{T} \int_{\Omega^{\prime}} \nabla_{x} \xi_{n} \cdot \phi d x d z d t=-\int_{0}^{T} \int_{\Omega^{\prime}} \xi_{n} \operatorname{div}_{x}(\phi) d x d z d t
$$

then, Lemma 1 provides:

$$
-\int_{0}^{T} \int_{\Omega^{\prime}} \xi_{n} \operatorname{div}_{x}(\phi) d x d z d t \rightarrow-\int_{0}^{T} \int_{\Omega^{\prime}} \xi \operatorname{div}_{x}(\phi) d x d z d t
$$

- We write the integral as follows:

$$
\int_{0}^{T} \int_{\Omega^{\prime}} \operatorname{div}_{x}\left(\xi_{n} D_{x}\left(\mathbf{u}_{n}\right)\right) \cdot \phi d x d z d t=-\int_{0}^{T} \int_{\Omega^{\prime}} \xi_{n} D_{x}\left(\mathbf{u}_{n}\right): \nabla \phi d x d z d t .
$$

Since $D_{x}\left(\mathbf{u}_{n}\right)=\frac{1}{2}\left(\nabla_{x} \mathbf{u}_{n}+\nabla_{x}^{t} \mathbf{u}_{n}\right)$, expanding the term in the last integral gives:

$$
\begin{aligned}
& -\int_{0}^{T} \int_{\Omega_{\Omega^{\prime}}} \xi_{n} D_{x}\left(\mathbf{u}_{n}\right): \nabla_{x} \phi d x d z d t \\
& =\frac{1}{2} \int_{0}^{T^{T}} \int_{\Omega^{\prime}}\left(\xi_{n} \mathbf{u}_{n} \cdot \Delta_{x} \phi+\nabla_{x} \phi \nabla_{x}\left(\sqrt{\xi_{n}}\right) \cdot \sqrt{\xi_{n}} \mathbf{u}_{n} d x d z d t\right. \\
& +\frac{1}{2} \int_{0}^{T} \int_{\Omega^{\prime}}\left(\xi_{n} \mathbf{u}_{n} \cdot \operatorname{div}_{x}\left(\nabla_{x}^{t} \phi\right)+\nabla_{x}^{t} \sqrt{\xi_{n}} \cdot \nabla_{x} \phi \cdot \sqrt{\xi_{n}} \mathbf{u}_{n}\right) d x d z d t .
\end{aligned}
$$


From Estimates (41), the sequence $\nabla_{x} \sqrt{\xi_{n}}$ weakly converges, and using Lemma 1, Lemma 3 and 4, we obtain:

$$
\begin{aligned}
& \frac{1}{2} \int_{0}^{T} \int_{\Omega^{\prime}}\left(\xi_{n} \mathbf{u}_{n} \cdot \Delta_{x} \phi+\nabla_{x} \phi \nabla_{x}\left(\sqrt{\xi_{n}}\right) \cdot \sqrt{\xi_{n}} \mathbf{u}_{n} d x d z d t\right. \\
&+\frac{1}{2} \int_{0}^{T} \int_{\Omega^{\prime}}\left(\xi_{n} \mathbf{u}_{n} \cdot \operatorname{div}_{x}\left(\nabla_{x}^{t} \phi\right)+\nabla_{x}^{t} \sqrt{\xi_{n}} \cdot \nabla_{x} \phi \cdot \sqrt{\xi_{n}} \mathbf{u}_{n}\right) d x d z d t \rightarrow \\
& \quad \frac{1}{2} \int_{0}^{T} \int_{\Omega^{\prime}}\left(\xi \mathbf{u} \cdot \Delta_{x} \phi+\nabla_{x} \phi \nabla_{x}(\sqrt{\xi}) \cdot \sqrt{\xi} \mathbf{u} d x d z d t\right. \\
&+\frac{1}{2} \int_{0}^{T} \int_{\Omega^{\prime}}\left(\xi \mathbf{u} \cdot \operatorname{div}_{x}\left(\nabla_{x}^{t} \phi\right)+\nabla_{x}^{t} \sqrt{\xi} \cdot \nabla_{x} \phi \cdot \sqrt{\xi} \mathbf{u}\right) d x d z d t .
\end{aligned}
$$

Hence

$$
-\int_{0}^{T} \int_{\Omega^{\prime}} \xi_{n} D_{x}\left(\mathbf{u}_{n}\right): \nabla_{x} \phi d x d z d t \rightarrow-\int_{0}^{T} \int_{\Omega^{\prime}} \xi D_{x}(\mathbf{u}): \nabla_{x} \phi d x d z d t .
$$

- We have straightforwardly

$$
\int_{0}^{T} \int_{\Omega^{\prime}} \partial_{z}^{2}\left(\xi_{n} \mathbf{u}_{n}\right) \cdot \phi d x d z d t \rightarrow \int_{0}^{T} \int_{\Omega^{\prime}} \xi_{n} \mathbf{u}_{n} \cdot \partial_{z}^{2}(\phi) d x d z d t .
$$

Using Lemma 3 provides the following convergence:

$$
\int_{0}^{T} \int_{\Omega^{\prime}} \xi_{n} \mathbf{u}_{n} \cdot \partial_{z}^{2}(\phi) d x d z d t \rightarrow \int_{0}^{T} \int_{\Omega^{\prime}} \xi \mathbf{u} \cdot \partial_{z}^{2}(\phi) d x d z d t
$$

- The convergence of the integral

$$
\int_{0}^{T} \int_{\Omega^{\prime}} r \xi_{n}\left|\mathbf{u}_{n}\right| \mathbf{u}_{n} \cdot \phi d x d z d t \rightarrow \int_{0}^{T} \int_{\Omega^{\prime}} r \xi|\mathbf{u}| \mathbf{u} \cdot \phi d x d z d t
$$

is obtained by Lemma 4, and finishes the proof of Theorem 2 .

\subsubsection{Proof of Theorem 1}

Following Ersoy et al. [6], to finish the proof of Theorem 1 we consider a sequence $\left(\xi_{n}, \mathbf{u}_{n}, w_{n}\right)$ of weak solution of System (19). All obtained estimates in steps 3.2.2-3.2.6 hold if we replace $\xi_{n}$ by $\rho_{n}$ and $w_{n}$ by $v_{n}$, since

$$
\rho(t, x, y)=\xi(t, x) e^{-y} \text { and } w(t, x, z)=v(t, x, y) e^{-y}
$$

where $\frac{d}{d y} z=e^{-y}$. Moreover, by the change of variables $z=1-e^{-y}$ in integrals, we have the following properties:

- $\|\rho\|_{L^{2}(\Omega)}=\alpha\|\xi\|_{L^{2}\left(\Omega^{\prime}\right)}$, 
- $\left\|\nabla_{x} \rho\right\|_{L^{2}(\Omega)}=\alpha\left\|\nabla_{x} \xi\right\|_{L^{2}\left(\Omega^{\prime}\right)}$,

- $\left\|\partial_{y} \rho\right\|_{L^{2}(\Omega)}=\alpha\|\xi\|_{L^{2}\left(\Omega^{\prime}\right)}$

where

$$
\alpha=\int_{0}^{1-e^{-1}}(1-z) d z<+\infty
$$

We deduce then,

$$
\|\rho\|_{W^{1,2}(\Omega)}=\alpha\|\xi\|_{W^{1,2}\left(\Omega^{\prime}\right)}
$$

which provides

$$
\rho \in L^{\infty}\left(0, T ; W^{1,2}(\Omega)\right)
$$

and

$$
\partial_{t} \rho \in L^{2}\left(0, T ; L^{2}(\Omega)\right) .
$$

Again, by the change of variable in integrals, the fact that $v \in L^{2}\left(0, T ; L^{2}(\Omega)\right)$ is obtained from the inequality:

$$
\begin{aligned}
\|v\|_{L^{2}(\Omega)} & =\int_{\Omega_{x}} \int_{0}^{1}|v(t, x, y)|^{2} d y d x \\
& =\int_{\Omega_{x}^{\prime}} \int_{0}^{1-e^{-1}}\left(\frac{1}{1-z}\right)^{3}|w(t, x, z)|^{2} d z d x \\
& <e^{3}\|w\|_{L^{2}\left(\Omega^{\prime}\right)} .
\end{aligned}
$$

Finally, all estimates on $\mathbf{u}$ remaining true, Theorem 1 is proved.

\section{Perspectives}

In this paper, we have presented a Compressible Primitive Equations where viscosities are anisotropic and density dependent. We have established a stability result for weak solutions by introducing a useful change of variable. The question of the existence of weak solutions for these equations remains an open question. However, with the obtained estimations, it may be possible to construct an approximate sequence of solutions, as Faedo-Galerkin approach and to adapt the technique presented by Vargant et al. [13]. Although, their models does not take into account the anisotropy and the dynamical viscosity is constant, useful additional estimates can be derived, particularly, to show that the density is bounded. The work is actually in progress.

\section{Acknowledgment}

The first author was supported by the ERC Advanced Grant FP7- 246775 NUMERIWAVES. 


\section{References}

[1] D. Bresch. Shallow-water equations and related topics. In Handbook of differential equations: evolutionary equations. Vol. V, Handb. Differ. Equ., pages 1-104. Elsevier/North-Holland, Amsterdam, 2009.

[2] D. Bresch and B. Desjardins. Some diffusive capillary models of Korteweg type. C. R. Acad. Sciences, 332(11):881-886, 2004.

[3] D. Bresch, B. Desjardins, and D. Gérard-Varet. On compressible NavierStokes equations with density dependent viscosities in bounded domains. J. Math. Pures Appl. (9), 87(2):227-235, 2007.

[4] D. Bresch, B. Desjardins, J.-M. Ghidaglia, and E. Grenier. Mathematical properties of the basic two fluid model. to appear in Arch. Rat. Mech. Anal., 2007.

[5] G. Buntebarth. Zur entwicklung des begriffes geophysik. Abhandlungen der Braunschwiegischen Wissenschaftlichen Gesellschaft, 32:95-109, 1981.

[6] M. Ersoy and T. Ngom. Existence of a global weak solution to one model of compressible primitive equations. Submitted, available at http:// hal. archives-ouvertes. fr/hal-00487370/fr/, 2010.

[7] B. V. Gatapov and A. V. Kazhikhov. Existence of a global solution to one model problem of atmosphere dynamics. Siberian Mathematical Journal, 46(5):805-812, 2005.

[8] N. E. Kochin. On simplification of the equations of hydromechanics in the case of the general circulation of the atmosphere. Trudy Glavn. Geofiz. Observator., 4:21-45, 1936.

[9] J.L. Lions, R. Temam, and S. Wang. New formulations for the primitive equations for the atmosphere and applications. Nonlinearity, 5:237-288, 1992.

[10] A. Mellet and A. Vasseur. On the barotropic compressible navier-stokes equations. Comm. Partial Differential Equations, 32(1-3):431-452, 2007.

[11] J. Pedlowski. Geophysical Fluid dynamics. 2nd Edition, Springer-Verlag, New-York, 1987.

[12] R. Temam and M. Ziane. Some mathematical problems in geophysical fluid dynamics. North-Holland, Amsterdam, 2004.

[13] V. A. Vaŭgant and A. V. Kazhikhov. On the existence of global solutions of two-dimensional Navier-Stokes equations of a compressible viscous fluid. Sibirsk. Mat. Zh., 36(6):1283-1316, ii, 1995. 